\title{
Factors influencing patient willingness to participate in genetic research after a myocardial infarction
}

\author{
David E Lanfear ${ }^{1 *}$, Philip G Jones ${ }^{2}$, Sharon Cresci ${ }^{3}$, Fengming Tang ${ }^{2}$, Saif S Rathore ${ }^{4}$ and John A Spertus ${ }^{2}$
}

\begin{abstract}
Background: Achieving 'personalized medicine' requires enrolling representative cohorts into genetic studies, but patient self-selection may introduce bias. We sought to identify characteristics associated with genetic consent in a myocardial infarction (MI) registry.

Methods: We assessed correlates of participation in the genetic sub-study of TRIUMPH, a prospective MI registry ( $\mathrm{n}$ $=4,340$ ) from 24 US hospitals between April 2005 and December 2008. Factors examined included extensive sociodemographics factors, clinical variables, and study site. Predictors of consent were identified using hierarchical modified Poisson regression, adjusting for study site. Variation in consent rates across hospitals were quantified by the median rate ratio (MRR).

Results: Most subjects consented to donation of their genetic material $(n=3,484 ; 80 \%)$. Participation rates varied greatly between sites, from $40 \%$ to $100 \%$. After adjustment for confounding factors, the MRR for hospital was 1.22 (95\% confidence interval (Cl) 1.11 to 1.29). The only patient-level factors associated with consent were race (RR 0.93 for African Americans versus whites, 95\% Cl 0.88 to 0.99 ) and body mass index (RR 1.03 for $\mathrm{BMI} \geq 25,95 \% \mathrm{Cl} 1.01$ to 1.06$)$.

Conclusion: Among patients with an $\mathrm{Ml}$ there were notable differences in genetic consent by study site, but little association with patient-level factors. This suggests that variation in the way information is presented during recruitment, or other site factors, strongly influence patients' decision to participate in genetic studies.
\end{abstract}

\section{Background}

As genetic research becomes more common and genetic factors are studied as a means for improving risk stratification and treatment, it is essential that participating subjects are representative of the general population of patients from which they are recruited. However, genetic research often attains lower participation rates compared with non-genetic studies [1]. Failure to recruit eligible subjects may also introduce selection biases into genetic studies, potentially jeopardizing both internal and external validity. Existing studies addressing this issue have revealed participation rates for genetic studies ranging from $21 \%$ to $99 \%$ [2-5]. This variability depends on many factors, including the disease under study [6],

\footnotetext{
* Correspondence: dlanfea1@hfhs.org

${ }^{1}$ Henry Ford Hospital, Heart and Vascular Institute, Detroit, Michigan, 48202, USA

Full list of author information is available at the end of the article
}

circumstances in which the patient is recruited [5], as well as a variety of patient characteristics that may impact patients' willingness to participate, including race $[7,8]$, education $[9,10]$, and gender $[3,7,8,11]$.

The existing literature has limited data regarding the genetic participation of patients with acute illnesses, which are required to study common cardiovascular diseases such as myocardial infarction (MI). First, some of the larger published studies are based upon opinion surveys (that is, asking whether the subject would be willing to participate in a theoretical genetic study) $[2,10,12]$. While these are important to help illuminate subjects' decision-making processes, subjects considering actual sample donation may behave differently when faced with the reality of undergoing blood/tissue collection, the potential risk of a confidentiality breach, or other real or perceived consequences of genetic analyses. Among studies that did involve actual donation and 
storage of samples, few have included large numbers of patients enrolled during the course of acute illness, which may also affect participation rates. For example, dissimilar participation rates have been reported for population-based studies compared to hospital-based cohorts [5]. Further clouding the literature is that most genetic studies have not adequately described populations from which samples were recruited, precluding an assessment of participation bias and potentially affecting internal and external validity [5]. The only study we are aware of examining genetic participation rates in an MI registry found significant clinical differences between participants and non-participants [13], calling into question the external validity of this study. A better understanding of the variability in patients' willingness to participate in genetic studies of acute cardiovascular disease is needed to assess for selection biases and to identify opportunities to improve participation and optimize the generalizability of such studies.

To address this existing knowledge gap, we examined characteristics associated with participation in a genetic sub-study within a large multi-center registry of MI patients. TRIUMPH (Translational Research Investigating disparities in Myocardial infarction Patients' Health status) is a multi-center study of MI patients at 24 US centers spread across the country and representing urban, suburban, academic, and community hospitals. A principal goal of this study was to assess genetic and pharmacogenomic factors associated with post-MI outcomes. Each enrolled patient was invited, but not required, to contribute DNA for genetic study. Participating hospitals were from diverse regions, including academic and community centers, a broad spectrum of economic and racial populations, as well as urban and rural locations. As such, TRIUMPH provided an ideal opportunity to examine patient factors associated with participation in genetic studies. Specifically, we sought to identify factors associated with genetic study participation, and to examine any differences between participants and those who chose not to donate their genetic material, in the hopes that these insights could improve recruitment in future studies.

\section{Materials and methods Participants}

All data and analyses presented here are part of the TRIUMPH study, a prospective registry of patients with acute MI from 24 hospitals across the United States (listed in Acknowledgements). The study was Institutional Review Board approved at all participating sites, and written informed consent was obtained from all participants. All patients who entered the registry were also offered participation in the genetic sub-study; however, this was not mandatory (that is, patients could participate in the registry without contributing DNA). A Federal Certificate of Confidentiality was obtained to further protect the confidentiality of patients' information and this was disclosed to patients in the informed consent document. Patients were enrolled from April 2005 to December 2008.

\section{Data collection}

For each patient in the parent study, detailed clinical and treatment characteristics were collected by chart abstraction and interview. Trained data collectors at each site participated in the acquisition of requisite data. Factors examined for association with genetic study participation included the socio-demographic, financial, social support, medical literacy, health status, depressive symptoms, clinical variables listed in Table 1 and enrollment site. All psychosocial and health status characteristics were quantified using standardized instruments, as previously described for the PREMIER study [14].

All study staff underwent similar training at in-person meetings. Ongoing data collection issues were addressed through monthly conference calls. There were similar staffing ratios (per-recruited patient) across sites. Templates for informed consent documents and educational pamphlets about the study were provided and used or modified by each site.

\section{Statistical analyses}

Patients were divided into two groups based on whether they consented to donate their genetic material (DNA) for storage and study, or not. Patient characteristics were compared using Chi-square tests for categorical variables and $t$-tests for continuous ones. The likelihood of consent was modeled using hierarchical regression that included a random effect for hospital. Because the consent rate was high, we estimated rate ratios (RRs) directly (that is, instead of estimating odds ratios) by using a modified Poisson regression model with robust standard errors [15]. For multivariable models, we initially included characteristics thought $a$ priori to be associated with participating in the genetic sub-study. These included age, race, gender, education, finances, social support, symptom severity, and hospital. In order to assess for potentially important patient characteristics, we also included the characteristics from Table 1 that showed univariate association with participation $(P<$ $0.05)$ in the multivariable models. Variation in consent rates between hospitals was quantified by the median rate ratio (MRR), which estimates the average relative difference in likelihood of two hypothetical patients, with identical covariates, consenting if enrolled at two different hospitals. Site participation rates are shown as smoothed estimates, which are a weighted average of the hospital's individual rate and the overall rate for the 


\begin{tabular}{|c|c|c|c|}
\hline & Consen & DNA & \\
\hline & Yes $(n=3,484)$ & No $(n=856)$ & $P$-value \\
\hline Demographics & & & \\
\hline Age & $58.9 \pm 12.2$ & $59.8 \pm 12.7$ & 0.038 \\
\hline White/Caucasian race & $2,342(67.4 \%)$ & $573(67.3 \%)$ & 0.981 \\
\hline Male & $2,347(67.4 \%)$ & $551(64.4)$ & 0.095 \\
\hline Language & & & 0.293 \\
\hline English & $3,353(98.4 \%)$ & $823(97.9 \%)$ & \\
\hline Spanish & $55(1.6 \%)$ & $18(2.1 \%)$ & \\
\hline Missing & 76 & 15 & \\
\hline Ethnicity & & & 0.870 \\
\hline Hispanic/latino & $217(6.4 \%)$ & $54(6.6 \%)$ & \\
\hline Non-hispanic/latino & $3,175(93.6 \%)$ & $770(93.4 \%)$ & \\
\hline Unknown & 92 & 32 & \\
\hline Low social support & $612(18.3 \%)$ & $109(13.1 \%)$ & $<0.001$ \\
\hline Missing & 133 & 23 & \\
\hline REALM-R score $\leq 6$ & $844(28.5 \%)$ & $170(28.1 \%)$ & 0.823 \\
\hline NA, missing, or unknown & 423 & 250 & \\
\hline Socio-economic status & & & \\
\hline Completed high school & $2,764(79.8 \%)$ & $656(76.9 \%)$ & 0.058 \\
\hline History of avoiding medical care due to cost & $904(26.5 \%)$ & $184(21.7 \%)$ & 0.004 \\
\hline End-of-month financial situation & & & $<0.001$ \\
\hline Some money left over & $1,380(40.4 \%)$ & $397(47.3 \%)$ & \\
\hline Just enough to make ends meet & $1,297(37.9 \%)$ & $295(35.1 \%)$ & \\
\hline Not enough to make ends meet & $741(21.7 \%)$ & $148(17.6 \%)$ & \\
\hline Medical history & & & \\
\hline BMI & $29.8 \pm 10.2$ & $29.0 \pm 6.5$ & 0.025 \\
\hline Chronic heart failure & $302(8.7 \%)$ & $70(8.2 \%)$ & 0.646 \\
\hline Dyslipidemia & $1,721(49.4 \%)$ & 407 (47.5\%) & 0.332 \\
\hline Hypertension & $2,318(66.5 \%)$ & $575(67.2 \%)$ & 0.722 \\
\hline Prior Ml & $710(20.4 \%)$ & $202(23.6 \%)$ & 0.038 \\
\hline Cancer & $250(7.2 \%)$ & $62(7.2 \%)$ & 0.946 \\
\hline Diabetes & $1,068(30.7 \%)$ & $268(31.3 \%)$ & 0.710 \\
\hline Presentation & & & \\
\hline Final Ml diagnosis & & & 0.568 \\
\hline STEMI & $1,475(42.3 \%)$ & $383(44.7 \%)$ & \\
\hline NSTEMI & $1,979(56.8 \%)$ & $465(54.3 \%)$ & \\
\hline BBB/uncertain type & $7(0.2 \%)$ & $2(0.2 \%)$ & \\
\hline Patient not diagnosed with Ml & $23(0.7 \%)$ & $6(0.7 \%)$ & \\
\hline Peak troponin & $29.3 \pm 76.6$ & $25.6 \pm 58.1$ & 0.187 \\
\hline Medications (arrival and discharge) & & & \\
\hline Aspirin on arrival & $1,431(41.1 \%)$ & $353(41.2 \%)$ & 0.930 \\
\hline Beta blocker at DC & $3,109(89.7 \%)$ & $776(91.4 \%)$ & 0.132 \\
\hline Thienopyridine on Arrival & $433(12.4 \%)$ & $113(13.2 \%)$ & 0.541 \\
\hline Statin at DC & $3,030(87.4 \%)$ & 744 (87.6\%) & 0.852 \\
\hline
\end{tabular}


Table 1 Patient characteristics in genetic sub-study participants versus non-participants (Continued)

\begin{tabular}{|c|c|c|c|}
\hline \multicolumn{4}{|l|}{ Processes of care } \\
\hline In-hospital cardiac catheterization & $3,222(92.5 \%)$ & 777 (90.8\%) & 0.096 \\
\hline In-hospital revascularization & $2,498(71.7 \%)$ & $618(72.2 \%)$ & 0.772 \\
\hline Enrolled in other study & $326(9.4 \%)$ & $70(8.2 \%)$ & 0.283 \\
\hline Length of stay & $5.6 \pm 6.4$ & $6.0 \pm 8.90$ & 0.207 \\
\hline \multicolumn{4}{|l|}{ Health Status } \\
\hline SAQ Quality of Life score & $62.2 \pm 23.6$ & $67.5 \pm 23.3$ & $<0.001$ \\
\hline SAQ Angina Stability score & $43.8 \pm 21.8$ & $47.1 \pm 20.6$ & $<0.001$ \\
\hline SAQ Physical Limitation score & $85.0 \pm 22.6$ & $88.4 \pm 19.6$ & $<0.001$ \\
\hline SF-12v2 Mental Component score & $49.6 \pm 11.5$ & $50.0 \pm 11.6$ & 0.411 \\
\hline SF-12v2 Physical Component score & $42.0 \pm 12.4$ & $42.8 \pm 12.5$ & 0.094 \\
\hline PHQ-9 depression severity & & & $<0.001$ \\
\hline Not clinically depressed & $1,763(54.4 \%)$ & $539(65.7 \%)$ & \\
\hline Mild depression & $831(25.6 \%)$ & $170(20.7 \%)$ & \\
\hline Moderate depression & $376(11.6 \%)$ & $72(8.8 \%)$ & \\
\hline Moderately severe depression & $191(5.9 \%)$ & $27(3.3 \%)$ & \\
\hline Severe depression & $81(2.5 \%)$ & $12(1.5 \%)$ & \\
\hline Missing & 242 & 36 & \\
\hline GRACE 6 m Mortality Risk score & $100.0 \pm 29.81$ & $103.0 \pm 31.1$ & 0.008 \\
\hline
\end{tabular}

Baseline patient characteristics are listed in the left-most column, with the quantities for those that participated in the genetic study, those that did not, and the $P$-value for difference between the two in the subsequent three columns. Categorical variables are shown as the number of subjects with that characteristic, followed by the proportion this represents (percentage) in parentheses. For variables that have subcategories, each subcategory and the number and proportion of subjects in that group is shown. Continuous variables are shown as the mean \pm the standard deviation. Categorical variables were compared using chi-square or Fisher's exact test. Continuous variables were compared using Student's t-test. BBB, bundle branch block; BMI, body mass index; DC; GRACE, Global Registry of Acute Coronary Events; NA, not applicable; NSTEMI, non-ST elevation myocardial infarction; PHQ, Patient Health Questionnaire; SAQ, Seattle Angina Questionaire; SF, Short Form; STEMI, ST elevation myocardial infarction.

entire cohort, where the weight given to an individual hospital is roughly proportional to their sample size. Smoothing was used in order to take into account the fact that some hospitals have small sample sizes and thus more uncertainty around their true rate.

Approximately $16.1 \%$ of patients had missing covariate data $(13.8 \%$ were missing one value, $1.8 \%$ were missing two values, and $0.5 \%$ were missing three or more values; the highest missing rate for any single variable (Patient Health Questionnaire (PHQ) depression score) was $6.4 \%$. Missing covariate data were imputed with multiple imputation using IVEwareE [16]. All analyses were performed in SAS version 9.1.3 (SAS Institute, Cary, North Carolina, USA), and R, version 2.7.0 (Foundation for Statistical Computing, Vienna, Austria).

\section{Results}

A total of 4,340 patients were enrolled in the study. Of these, 3,484 (80\%) consented to donate their DNA for study. Clinical and socio-demographic characteristics among genetic sub-study participants versus non-participants are summarized in Table 1 . Several socio-demographic factors differed between participants and nonparticipants in unadjusted analyses, including measures of social support, literacy, education, financial hardship, and smoking status. Among clinical variables, health status, body mass index (BMI), history of MI, history of stroke, and receiving beta-blockers on arrival each had univariate associations with genetic consent. The genetic participation rate varied across enrolling sites, ranging from $40 \%$ to $100 \%$. Smoothed estimates of site participation rates derived from the random effects model are shown in Figure 1.

A multivariable modified Poisson model was then constructed to test for factors associated with consenting to genetic testing (Figure 2). The only factors independently associated with participation were African American race, enrollment site, and BMI. African American race was associated with a $7 \%$ lower rate of consenting to genetic study compared with white patients (RR 0.93 ; 95\% confidence interval (CI) 0.88 to 0.99 ). Higher BMI $(\geq 25)$ was marginally associated with a slightly higher participation with RR of 1.03 (95\% CI 1.01 to 1.06$)$. Several other factors were of borderline significance, including PHQ-9 score (RR 1.02 for every 5 points; $95 \%$ CI 1.00 to 1.05 ) and chronic lung disease (RR 1.04; 95\% CI 1.00 to 1.08). By far, the strongest factor associated with participating in the genetic study was enrollment site. The MRR was 1.22 (95\% CI 1.11 to 1.29), suggesting that an identical patient presenting at one hospital would, on average, have a nearly 1 in 4 greater likelihood of participating in a genetic study 


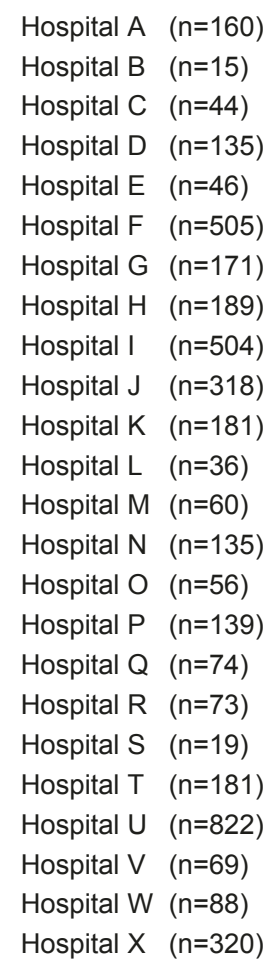

Figure 1 Genetic consent rates by hospital. Each hospital is labeled by letters A to H (vertical axis). Each dot and line represents the proportion of subjects at the site that consented to genetic sub-study enrollment. The central dot shows the point estimate of the site rate (percentage) generated from the random effects models. The lines extending from the dot represent the $95 \%$ confidence interval.

than if that same patient had presented to a different TRIUMPH hospital.

\section{Discussion}

We sought to define characteristics associated with participation in a genetic sub-study of a large acute MI registry. We found that the vast majority of patients chose to participate in genetic testing (around 80\%), with few differences between those who did and did not agree to donate DNA. Although we found race to be mildly associated with patients' willingness to participate in genetic studies, other factors such as gender and education level were not. Most importantly, the strongest predictor of participation in the genetic sub-study was hospital site, with wide variability seen in rates across sites.

Reduced genetic participation among racial minorities is a particularly critical issue since racial disparities in health outcomes are high-priority research topics, and the genetic versus non-genetic components of health disparities need to be better elucidated. Higher rates of participation in genetic studies among white patients, as compared with African Americans, have been previously described $[4,7,8]$. A lower likelihood of African
American participation in medical research generally has also been well described, with lack of trust or confidence in the researchers being one important factor [17]. Similarly, trust is one of the most often cited mediating factors for participation in genetic studies [2], and this is also the case in studies specifically focusing upon racial differences in genetic research; patient concerns about confidentiality were a consistent reason for choosing not to participate $[12,18]$. In our study, African Americans were $7 \%$ less likely to participate than whites, a modest difference in participation rates. While further qualitative studies may help illuminate the mechanism, awareness of this potential selection bias is important during study enrollment so that under-representation of racial minorities can be minimized. Making every effort to establish trust and rapport with subjects, as well as confidence in the research team and their confidentiality protections, may help reduce refusal rates.

To our knowledge, the association of genetic consent with BMI has not been previously reported, and the magnitude of the association is of questionable clinical significance. Given the number of possible predictors included in this study, this association may be spurious. 


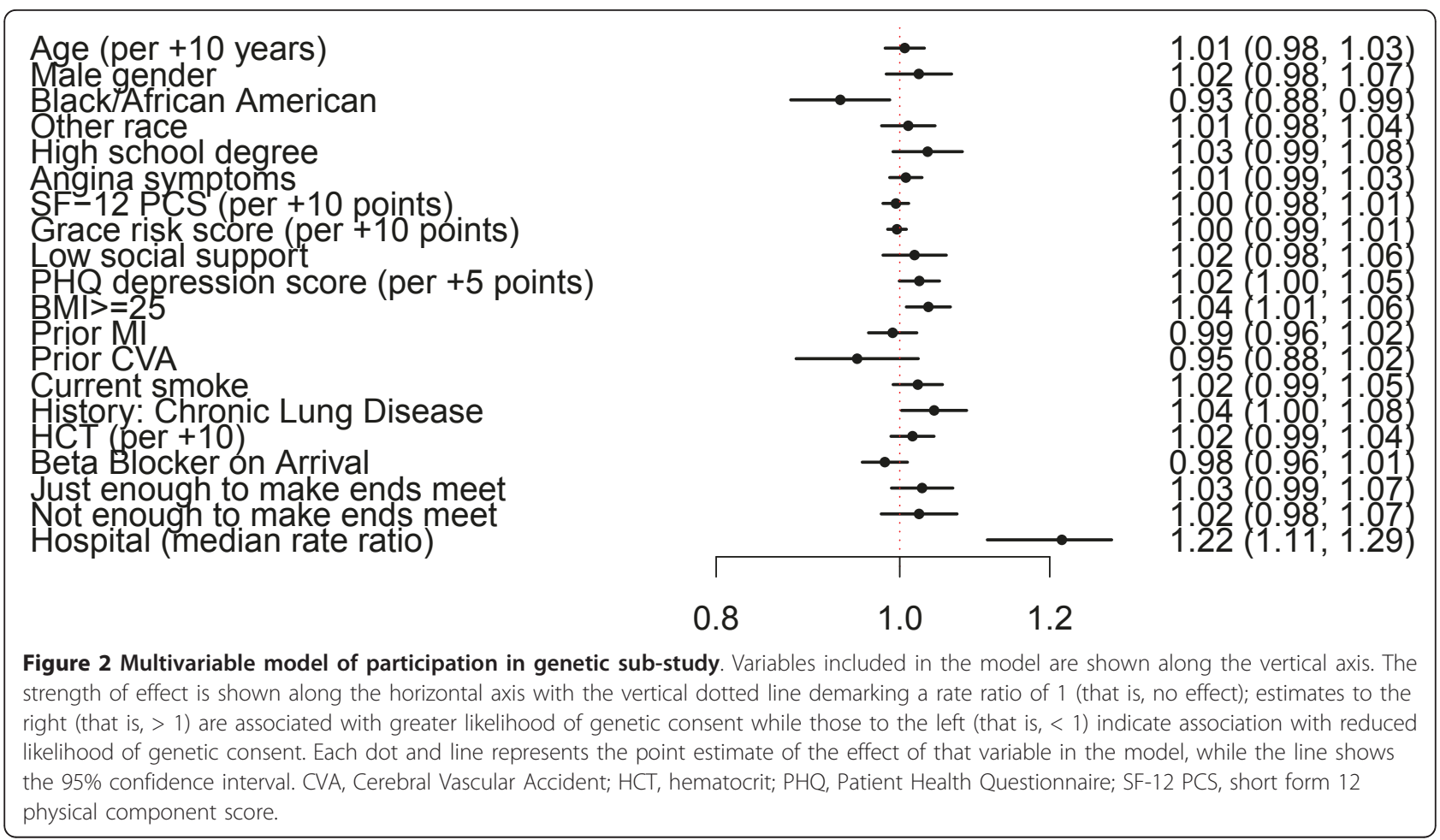

Confirmation of this finding in an independent cohort is needed and, if consistent findings are observed, then qualitative research could be used to better understand the potential mechanism of this association. In contrast to previous studies, our data did not show any other patient level characteristics to be significantly associated with patients' willingness to consent to genetic testing.

Some additional aspects of our data are worth noting. First, our study examined acutely ill hospitalized patients, while most previous studies were outpatient or population-based. We found rates of participation roughly similar to previous studies of patients that had already consented to non-genetic research $[4,19,20]$. The only other published genetic MI registry addressing participation rates [13], identified clinical selection biases, but these were not confirmed in ours. In contrast, our data demonstrated that patients consenting to genetic participation were overall quite similar to those who chose not to participate across a wide range of clinical factors. This difference may be due to the fact that ours is a multi-center cohort, as opposed to the single-center experience of the previous study. Given the importance of recruitment site in our study, there may have been unique characteristics of that site that influenced their findings. Nevertheless, it is critically important that genetic association studies explicitly quantify potential selection biases of the participating cohort compared with the parent population to whom the conclusions will be applied. The similarity of our genetic versus non-genetic patients supports the external validity of the future genetic analyses planned for these data.

Most importantly, we were able to clearly identify that site of recruitment was the most important factor associated with participation. While the mechanism can not be stated with certainty, this most likely reflects variations between centers in the presentation style of individual study coordinators, their motivation to recruit into the genetic study, ability to establish rapport and trust, or their ability to provide complete information to patients' satisfaction and comfort. If this is true, the marked variation across sites indicates an important opportunity, through better training and standardization, to improve enrollment processes in future studies. Ensuring high-quality and consistent consent processes should reduce variability in consent rates and may also provide overall enhanced participation in genetic association studies. This is highly desirable in order to minimize the potential for bias and enhance generalizability. Although specific training regarding genetic enrollment was done at the beginning of our study, changes in study coordinators and shifts in their responsibilities may have limited the effectiveness of the initial standardization for DNA acquisition across sites. Moreover, testing, through role-playing, coordinators' skills in obtaining informed consent are important steps for future studies to consider. We further suggest that future studies provide ongoing assessments of the rates of genetic consents at each center to rapidly identify 
differences between site participation rates so that proactive education of site coordinators can occur throughout the study. These data also underscore the importance of close collaborations between investigators, coordinators, Institutional Review Boards and others involved in genetic studies to optimize communication with subjects, assess their comprehension, and to provide strong protections (for example, confidentiality) that can maximize patient comfort with, and participation in, genetic research.

Our findings should be interpreted in the context of the following potential limitations. First, we can not completely exclude the possibility that unidentified variation in patient characteristics between sites may have led to residual confounding of the observed differences in participation rates. Specifically, there could theoretically be regional differences in patient attitudes towards genetic study that influence participation decisions that were not quantifiable from our extensive data collection, given that geographic region and enrollment site are highly correlated. Second, our data do not identify the mechanism underlying our observed associations, which would require additional qualitative studies to better understand determinants of patient decision-making.

\section{Conclusions}

Our multi-center study was able to engage $80 \%$ of patients to participate in genetic research at the time of their acute MI. Genetic participants were clinically similar to those who chose not to donate their genetic material. African American patients, as compared with white patients, had a slightly lower rate of genetic participation, but no other patient-level factors, including gender and education, were significantly associated with consent. While BMI was statistically associated with participation rates, the magnitude of the effect was small and this association has not been previously observed to our knowledge. Most importantly, the strongest factor associated with genetic consent was enrollment site. This suggests that differences in how study personnel interact with patients are a key determinant of their willingness to participate, and should be prospectively monitored in future studies to maximize participation rates in genetic investigations.

\section{Abbreviations}

BMl: body mass index; Cl: confidence interval; Ml: myocardial infarction; MRR: median rate ratio; PHQ: Patient Health Questionnaire; RR: rate ratio; TRIUMPH: Translational Research Investigating disparities in Myocardial infarction Patients' Health status.
}

\section{Acknowledgements}

This research was funded by the National Institutes of Health through the National Heart, Lung, and Blood Institute SCCOR in Diabetic Heart Disease
(P50HL077113). It was also supported in part by National Heart, Lung, and Blood Institute Career Development Award (K23HL085124; PI Lanfear). Mr Rathore is supported in part by CTSA Grant Number UL1 RR024139 from the National Institutes of Health's Center for Research Resources, a National Institute of General Medical Sciences Medical Scientist Training Program grant (5T32GM07205), and an Agency for Healthcare Research and Quality dissertation grant. Saint Luke's Mid America Heart Institute is the TRIUMPH Coordinating Center and members of the Cardiovascular Outcomes Research Consortium participating in this study included: Barnes Jewish Hospital/ Washington University, Saint Louis, MO - Richard Bach MD; Bridgeport Hospital, Bridgeport, CT - Stuart Zarich MD; Christiana Care Health System, Newark, DE - William Weintraub MD; Denver General Health System, Denver, CO - Frederick Masoudi MD MSPH, Edward Havranek MD; Duke University, Durham, NC - Karen Alexander MD, Eric Peterson MD MPH; Grady Health Systems/Emory University, Atlanta, GA - Susmita Parashar MD MPH MS, Viola Vaccarino MD PhD; Henry Ford Hospital, Detroit, MI - Aaron Kugelmass MD, David Lanfear MD; John H Stroger Jr Hospital of Cook County, Chicago IL Amit Amin MD, Sandeep Nathan MD, Russell Kelley MD; Leonard J Chabert Medical Center, Houma, LA - Lee Arcement MD MPH; MeritCare Medical System, Fargo ND - Walter Radtke MD, Thomas Haldis MD; Montefiore Medical Center, Bronx, NY - VS Srinivas MD; Presbyterian Hospital, Albuquerque, NM - Dan Friedman MD; Saint Luke's Mid America Heart Institute, Kansas City, MO - John Spertus MD MPH; Sentara Health System (both Sentara and Sentara Leigh Hospitals), Norfolk, VA - John E Brush Jr MD; Truman Medical Center and the University of Missouri - Kansas City, Kansas City, MO - Mukesh Garg MD, Darcy Green Conaway MD; Tufts-New England Medical Center, Boston MA - Jeffrey T Kuvin MD; University of Colorado Health System, Denver, CO - John Rumsfeld MD PhD, John Messenger MD; University of lowa, lowa City, IA - Phillip Horwitz MD; University of Michigan Health Systems, Ann Arbor, MI - Brahmajee Nallamothu MD MPH; University of Texas Southwestern, Dallas, TX - Darren McGuire MD MHSc; VA lowa City Health Care System, lowa City, IA - Phillip Horwitz MD; Virginia Commonwealth University, Richmond, VA - Michael C Kontos MD; Yale University/Yale-New Haven Hospital, New Haven, CT Harlan Krumholz MD.

\section{Author details}

${ }^{1}$ Henry Ford Hospital, Heart and Vascular Institute, Detroit, Michigan, 48202, USA. ${ }^{2}$ Mid-America Heart Inst, Kansas City, Missouri, 64134, USA. ${ }^{3}$ Washington University in St Louis, Department of Medicine, Division of Cardiology, St Louis, Missouri, 63108, USA. ${ }^{4}$ MD/PhD Program, Yale University School of Medicine, New Haven, Connecticut, 06510, USA.

\section{Authors' contributions}

DEL contributed to the study conception and design, data analysis, drafted the manuscript, and approves of the final manuscript. PGJ contributed to the acquisition of data, data analysis, critically revising the manuscript, and approves of the final manuscript. SC contributed to the data analysis and interpretation, critically revising the manuscript, and approves of the final manuscript. FT contributed to the acquisition of data, data analysis, critically revising the manuscript, and approves of the final manuscript. SSR contributed to the data analysis and interpretation, critically revising the manuscript, and approves of the final manuscript. JAS contributed to the study design, data analysis, drafting of the manuscript, and approves of the final manuscript.

\section{Competing interests}

The authors declare that they have no competing interests.

Received: 1 April 2011 Revised: 9 May 2011 Accepted: 15 June 2011 Published: 15 June 2011

\section{References}

1. Matsui $K$, Kita $Y$, Ueshima $H$ : Informed consent, participation in, and withdrawal from a population based cohort study involving genetic analysis. J Med Ethics 2005, 31:385-392.

2. Kettis-Lindblad A, Ring L, Viberth E, Hansson MG: Genetic research and donation of tissue samples to biobanks. What do potential sample donors in the Swedish general public think? Eur J Public Health 2006, 16:433-440.

3. Stewart-Knox BJ, Bunting BP, Gilpin S, Parr HJ, Pinhao S, Strain JJ, de Almeida MD, Gibney M: Attitudes toward genetic testing and 
personalised nutrition in a representative sample of European consumers. Br J Nutr 2009, 101:982-989.

4. Sterling R, Henderson GE, Corbie-Smith G: Public willingness to participate in and public opinions about genetic variation research: A review of the literature. Am J Public Health 2006, 96:1971-1978.

5. Meschia JF, Merino JG: Willingness of ischemic stroke patients to donate DNA for genetic research: A systematic review. J Stroke Cerebrovasc Dis 2003, 12:228-231.

6. Treloar SA, Morley KI, Taylor SD, Hall WD: Why do they do it? A pilot study towards understanding participant motivation and experience in a large genetic epidemiological study of endometriosis. Community Genet 2007, 10:61-71.

7. Ford BM, Evans JS, Stoffel EM, Balmana J, Regan MM, Syngal S: Factors associated with enrollment in cancer genetics research. Cancer Epidemiol Biomarkers Prev 2006, 15:1355-1359.

8. McQuillan GM, Pan Q, Porter KS: Consent for genetic research in a general population: an update on the National Health and Nutrition Examination Survey experience. Genet Med 2006, 8:354-360.

9. Skinner CS, Schildkraut JM, Calingaert B, Hoyo C, Crankshaw SS, Fish L, Susswein L, Jasper C, Reid L: Factors associated with African Americans' enrollment in a national cancer genetics registry. Community Genet 2008, 11:224-233.

10. Wang SS, Fridinger F, Sheedy KM, Khoury MJ: Public attitudes regarding the donation and storage of blood specimens for genetic research. Community Genet 2001, 4:18-26.

11. Crider KS, Reefhuis J, Woomert A, Honein MA: Racial and ethnic disparity in participation in DNA collection at the Atlanta site of the National Birth Defects Prevention Study. Am J Epidemiol 2006, 164:805-812.

12. Bogner HR, Wittink MN, Merz JF, Straton JB, Cronholm PF, Rabins PV, Gallo JJ: Personal characteristics of older primary care patients who provide a buccal swab for apolipoprotein E testing and banking of genetic material: The spectrum study. Community Genet 2004, 7:202-210

13. Arruda-Olson AM, Weston SA, Fridley BL, Killian JM, Koepsell EE, Roger VL: Participation bias and its impact on the assembly of a genetic specimen repository for a myocardial infarction cohort. Mayo Clin Proc 2007, 82:1185-1191.

14. Spertus JA, Peterson E, Rumsfeld JS, Jones PG, Decker C, Krumholz H: The prospective registry evaluating myocardial infarction: Events and recovery (PREMIER) - evaluating the impact of myocardial infarction on patient outcomes. Am Heart J 2006, 151:589-597.

15. Zou G: A modified poisson regression approach to prospective studies with binary data. Am J Epidemiol 2004, 59:702-706.

16. Raghunathan TE, Solenberger PW, Van Hoewyk J: Imputation and Variance Estimation Software - User Guide Ann Arbor, Michigan: IVEware; 2002.

17. Shavers VL, Lynch CF, Burmeister LF: Racial differences in factors that influence the willingness to participate in medical research studies. Ann Epidemiol 2002, 12:248-256.

18. Audrain J, Tercyak KP, Goldman P, Bush A: Recruiting adolescents into genetic studies of smoking behavior. Cancer Epidemiol Biomarkers Prev 2002, 11:249-252.

19. Levy D, Splansky GL, Strand NK, Atwood LD, Benjamin EJ, Blease S, Cupples LA, D'Agostino RB Sr, Fox CS, Kelly-Hayes M, Koski G, Larson MG, Mutalik KM, Oberacker E, O'Donnell CJ, Sutherland P, Valentino M, Vasan RS, Wolf PA, Murabito JM: Consent for genetic research in the Framingham Heart Study. Am J Med Genet A 2010, 152:1250-1256.

20. Melas PA, Sjoholm LK, Forsner T, Edhborg M, Juth N, Forsell Y, Lavebratt C Examining the public refusal to consent to DNA biobanking: empirical data from a Swedish population-based study. J Med Ethics 2010, 36:93-98.

doi:10.1186/gm255

Cite this article as: Lanfear et al:: Factors influencing patient willingness to participate in genetic research after a myocardial infarction. Genome Medicine 2011 3:39.

\section{Submit your next manuscript to BioMed Central and take full advantage of:}

- Convenient online submission

- Thorough peer review

- No space constraints or color figure charges

- Immediate publication on acceptance

- Inclusion in PubMed, CAS, Scopus and Google Scholar

- Research which is freely available for redistribution

Submit your manuscript at www.biomedcentral.com/submit 